\title{
Importance of the initial conditions and the time schedule in the Simulated Annealing
}

\author{
K. Shojaee ${ }^{1}$, H. Shakouri $\mathrm{G}^{2}$ and M. Behnam Taghadosi ${ }^{3}$ \\ A Mushy State SA for TSP
}

\begin{abstract}
It is a long time that the Simulated Annealing (SA) procedure is introduced as a nonderivative based optimization for solving NP-hard problems. Improvements from the original algorithm in the recent decade mostly concentrate on combining its initial algorithm with some heuristic methods. This is while modifications are rarely happened to the initial condition selection methods from which the annealing schedules starts or the time schedule itself. There are several parameters in the process of annealing the adjustment of which affects the overall performance. This paper focuses on the initial temperature and proposes a lower temperature with low energy to speed up the process, while using an auxiliary memory to buffer the best solution. Such an annealing indeed starts from a mushy state rather than a quite liquid molten material. The mushy state characteristics depends on the problem that SA is being applied to solve. In this paper the Mushy State Simulated Annealing (MSSA) is applied to the Traveling Salesman Problem (TSP). The mushy state may be obtained by some simple methods like crossover elimination. A very fast version of a Wise Traveling Salesman, who starts from a randomly chosen city and seeks for the nearest one as the next, is also applied to initiate SA by a low-energy-low-temperature state. This fast method results in quite accurate solutions compared to other recent novel methods.
\end{abstract}

Keywords

Combinatorial Optimization, Traveling Salesman, Initial Condition

1 Low-Power High-Performance Nanosystems Laboratory, School of Electrical and Computer Engineering, University of Tehran, Tehran, Iran (email:k.shojaee@ece.ut.ac.ir)

2 Industrial Engineering Department, University of Tehran, Tehran, Iran (email: hshakouri@ut.ac.ir)

${ }^{3}$ Mechatronics Laboratory (LIM), politecnico di Torino, Torino, Italy (email:mojtaba.behnam@polito.it) 


\section{Introduction}

Simulated Annealing (SA) is one of the earliest methods for derivative-free optimization such as Tabu Search (TS) [1]. Although it was introduced first to solve combinatorial discrete problems [2], it has recently shown a high attitude for solution of continuous problems as well [3]-[5]. SA is derived from physical behaviour of molten metals when the temperature is slowly falling to form a regular crystalline solid structure. There are two key parameters in the cooling process that determine how firm or amorphous will be the result for the metal in its frozen state. The first one is the initial temperature from which the cooling starts; and the second is the rate by which the temperature is falling.

Concerning the rate of decay, it should be low enough to allow the atoms in the molten metal to line them up and give enough time to form a crystal lattice with the minimum internal energy.

Evidently, a slow decay will lead to a long time for the solidifying process. To reduce the time, one may think of a low initial temperature. However, on the other hand, if the initial temperature is not high enough, atoms of the molten metal would not have enough freedom to rearrange their positions in a very regular minimum energy structure.

Although there are some theoretical limits and formulations to choose a proper cooling rate [6]-[22], there is not any deterministic criterion to set the initial pseudo-temperature in the literature. For instance, applying SA to the travelling salesman problem (TSP), one may set it to 0.5 and change by $10 \%$ at each step [9], while some other prefer 1000 reducing by a factor of 0.99 , i.e. $1 \%$ [10]. Moreover, the concept is a case dependent one and even may not fit to a bounded range, e.g. in some articles it is even initialized in a range from 0.001 to 100 [11].

There are a few research papers that suggest a formulation to relate the initial temperature to particular characteristics of the problem. Pao et al. considered an initial temperature such that the initial acceptance rate is about 70\% [12]. Feng-Tse Lin, et al. proposed an AnnealingGenetic approach and use the following formula [13]:

$T_{0}=\Delta E /($ Population Size/2),

where $\Delta E$ is the difference between the highest cost and the lowest cost found for the first generation of the randomly generated population.

Thompson and Bilbro set the initial temperature by defining a probability function for energy change in continuous problems. The probability of accepting a higher cost solution was set to 0.75 . Then, the following probability distribution is solved to find $T_{0}[14]$ :

$p=\exp (\Delta E / T)$,

where $\Delta E$ is the average cost of the random solutions plus its standard deviation.

Hao Chen et al. set the initial temperature such that the initial acceptance probability for an average uphill move is 0.97 [15].

Although SA algorithms are conceptually simple, finding optimal parameters such as initial temperature, the annealing schedule, the acceptance function parameters, etc., is by no means simple or straightforward. First of all, setting parameters for SA is problem dependent, and it is best accomplished through trial and error. Furthermore, many studies have demonstrated that SA algorithms are very sensitive to parameters, and their performances are largely dependent on fine tuning of the parameters [16]. The problem dependent nature of setting parameters for SA and its sensitivity to parameters limit the effectiveness and robustness of SA algorithms. SA possesses a formal proof of convergence to the global optima. This convergence proof relies on a very slow cooling schedule of setting the initial condition to a sufficiently large temperature and let it decay by $T_{k}=T_{0} /$ 
$\log (k)$, where $k$ is bound by the number of iterations[17]. While this cooling schedule is impractical, it identifies a useful trade-off where longer cooling schedules tend to lead to better quality solutions.

Also, the stochastic simulated annealing (SSA) [7] tends to find a global optimum if the annealing process is carried out sufficiently slowly. It means that SSA is able to find highquality solutions (global optima or near-global-optima), if the temperature is reduced exponentially but with a sufficiently small exponent. For many applications, this may mean prohibitively long relaxation time in order to find solutions of acceptable quality, and conversely, reasonably long periods of time may still result in poor solutions. Lipo Wang et al. have used chaotic neural networks to be combined with the best features of SSA and have shown the effectiveness of this new stochastic chaotic simulated annealing (SCSA) [18]. However there is not any especial idea on the initializing or the cooling schedule in this approach. Before, Yuyao He had applied a chaotic noise to a Hopfield neural network and had set the annealing process such that the chaotic noise gradually reduced. Hence, it was initially chaotic but eventually convergent, and, thus, had shown richer and more flexible dynamics [19].

In brief, we observe that there is a trade off between choosing a high initial temperature or choosing a low rate of cooling, and gaining a short processing time or finding the minimum energy structure.

Exactly similar to such a trade off exists when applying SA to any optimization problem such as TSP. Assuming the objective function of an optimization problem to be an energy function, and the initial guesses for the unknown variables to be the initial problem, the above mentioned trade off appears as shown by the following notation:

$\begin{array}{ll}\text { Initial temperature } \uparrow & \Rightarrow \text { Optimization time } \uparrow \\ \text { Initial temperature } \downarrow & \Rightarrow \text { Final energy } \uparrow \text { (Local minima) } \\ \text { Rate of cooling } \uparrow & \Rightarrow \text { Final energy } \uparrow \text { (Local minima) } \\ \text { Rate of cooling } \downarrow & \Rightarrow \text { Optimization time } \uparrow\end{array}$

It is easy to deduce that selection of a proper set of optimization parameters for SA itself is a multi-objective decision making (optimization) problem. In this paper we have discussed the first one, i.e. the initial temperature, and propose an approach to speed up the algorithm while obtaining accurate solutions for the chosen case study, which is TSP.

It is usual to select a very high temperature that provides a suitable initial condition with enough mobility for the atoms to move freely to new locations faraway enough in order to form as possible as minimum-energy structures. A certain criterion is to set it large enough that almost any trial point (state) will be acceptable.

This may cause the SA process to experiment new accepted points with even higher energy states. As the temperature decays, the probability to accept states that do not reduce the energy decreases.

Since the cooling process that starts from a high temperature in a liquid-like state is a time consuming, this paper proposes to start annealing from a state in a lower temperature with a lower internal energy. Such a state may be called a mushy state, rather than a liquid state. In such reduced temperatures with low energy, the ratio of acceptable states to the total trials may be less than $10 \%$, compared to that of usual high temperatures. 
After that the state is set to a lower energy state in a lower initial temperature, the annealing process can bring us the benefit of a faster local search and find the optimal state with the minimum energy. Starting from a very high temperature the metal should be cooled slowly, otherwise the atoms do not have time to orient themselves into a regular structure, but if the initial state is imposed to the atoms in a low energy low temperature, we can adjust the cooling rate to be faster.

Perhaps there are many optimization methods, even direct (random search methods) that can be applied as a prelude for SA. A simple algorithm that is used in this paper is to eliminate all intersecting paths in an initially selected random tour. A second simple method is also applied to show independence of the proposed method to the method that the initial tour is found.

The paper is organized in six sections. After this section we will have short introductions to both the SA and the TSP in sections 2 and 3. Section 4 describes how we chose the initial conditions and how schedule the annealing. The results obtained applying the proposed method are given in section 5, where we have compared the best, the worst and the average error in the final solution (if available) with some recent works. Finally, section 6 concludes the paper.

\section{A short overview on the SA}

Rather than giving a detailed description of SA, herein the fundamental terminology of SA is explained shortly [8][21]. The method consists of four main parts.

\subsection{Objective function}

An objective function $f($.) is a mapping from an input vector $x$ into a scalar $E$ :

$$
E=f(x)
$$

where each $x$ is assumed as a point in an input space. The SA is to sample the input space effectively to find an $x$ that minimizes $E$. The input vector may be the structure of the atoms and/or their movements limited to that structure, and $E$ may be the internal energy of the metal. In TSP, $x$ is the tour sequence and $E$ is the total cost (distance) of travelling.

\subsection{Generating function}

A generating function $g(.,$.$) specifies the probability density function of the difference$ between the current point and the next point to be visited. Specifically, $\Delta x\left(=x_{\text {new }}-x\right)$ is a random variable with probability density function $g(\Delta x, T)$, where $T$ is the pseudotemperature. If $E$ is the internal energy of the metal, $T$ is the real temperature, however, in TSP can be interpreted as percentage of the new points that can reduce the total cost. Clearly, if the number of intersecting paths in a tour $x$ is high, we can assume that the pseudo-temperature is high. Usually $g(.,$.$) is independent of the temperature. However, in$ conventional SA, also known as Boltzmann machines, the generating function is a Gaussian probability density function:

$$
g(\Delta x, T)=(2 \pi T)^{-n / 2} \exp \left[-|\Delta x|^{2} /(2 T)\right],
$$


where $n$ is the dimension of the space under exploration. The fatter tail of the Cauchy distribution gives the chance to explore new points in the space farther from the current point while searching the space.

For discrete or combinatorial optimization problems, like TSP, each $x$ is not necessarily an $n$ vector with unconstrained values. Instead, each $x$ is restricted to be one of $N$ points that comprise the solution space or the input space. Usually $N$ is very large but finite such that reduces probability of a time consuming search without any result. Since, adding randomly generated $\Delta x$ to a current point $x$ may not generate another legal point in the solution space, instead of using generating functions, a move set is usually defined to find the next legal point, denoted by $M(x)$. This creates the set of legal points available for exploration after $x$. Usually the move set, $M(x)$, is chosen in the sense that the objective function at any point of the move set, i.e. a set of neighbouring points $x+\Delta x$, will not differ too much from the objective function at $x$. The definition of the move set is problem dependent. For TSP there are at least three kinds of move sets that are defined and used by researchers: Inversion, Translation, and Switching [21]. An especial variant of inversion is the simple idea of Crossover elimination.

Once the move set is defined, $x_{\text {new }}$ is usually selected at random from the move set, such that all neighbouring points have an equal probability of being chosen. In this paper, we have fixed the move set to the inversion, which has shown better performance compared to the others.

\subsection{Acceptance function}

After that the objective is evaluated for a new point $x_{\text {new }}$, SA decides whether to accept or reject it based on the value of an acceptance function $h(.,$.$) . The most frequently used$ acceptance function is the Boltzmann probability distribution:

$$
h(\Delta E, T)=\frac{1}{1+\exp (\Delta E /(c T))}
$$

where $c$ is a constant, $T$ is the temperature, and $\Delta E$ is the energy difference between $x_{\text {new }}$ and $x$ :

$$
\Delta E=f\left(x_{\text {new }}\right)-f(x)
$$

Usually $x_{\text {new }}$ is accepted with probability $h(\Delta E, T)$. If $\Delta E$ is negative, SA tends to accept the new point to reduce the energy. Nevertheless, if $\Delta E$ is positive SA may also accept the new point and move to a higher energy state. It means, SA can move uphill or downhill; but the lower the temperature, the less likely to accept any significant upward change.

There are several alternatives for the acceptance function. A simple alternative with approximately the same behaviour is:

$$
h(\Delta E, T)=\exp \left(-\frac{\Delta E}{c T}\right)
$$


where there is no need to check for the sign of $\Delta E$. Instead, if $h(\Delta E, T)$ is greater than a uniformly distributed random number, the new point is accepted. A deterministic alternative method is to use Threshold Accepting, where $x_{\text {new }}$ is accepted just if $\Delta E<T$ [22].

\subsection{Annealing schedule}

An annealing or cooling schedule regulates how rapidly the temperature $T$ goes from high to low values, as a function of time or iteration counts. There are not so many works discussing the initial temperature selection or even the cooling schedule. Indeed, the exact interpretation of high and low and the specification of a good annealing schedule require certain problem-specific physical insight and/or trial-and-error. The easiest way of setting an annealing schedule is to decrease the temperature $T$ by a certain percentage at the $k^{\text {th }}$ iteration:

$$
T_{k+1}=a T_{k}
$$

where $0<a<1$ is an adjusting parameter. It is proved that a Boltzmann machine using the aforementioned generating function can find a global optimum of $f(x)$ if the temperature $T$ is reduced not faster than $T_{0} / \log (k)$ [17]. Researchers have used various cooling strategies, among which we choose the following:

$$
T_{k+1}=T_{k} / \log \left(k^{1 / D}\right),
$$

where $D$ is set to two.

\section{The Traveling Salesman Problem (TSP)}

The Travelling Salesman Problem (TSP) seems to be the most well-known typical NP-hard problem. Given a set of nodes and a set of weights specifying cost to travel between each two nodes, the optimal solution is to find a closed loop of the paths with minimal total weights in a finite complete graph.

Lets denote the set of "cities" in TSP as $C=\left\{c_{1}, c_{2}, \ldots, c_{n}\right\}$ in company with a matrix $D_{T}$ an element of which is called $d_{i j}$ that gives the distance or cost function (weight) for going from $t_{i}$, to $t_{j}$. In real problems, usually the coordinates of the cities are given, by which the matrix $D_{T}$ can be easily computed.

The path linking the two cities here is called a "link". A sequence of cities $C^{*}=\left[c_{s 1}, c_{s 2}, \ldots\right.$, $c_{s n}$ ] denotes a legal solution of TSP (the salesman must visit each city once and only once), where $\left\{s_{1}, s_{2}, \ldots, s_{n}\right\}$ is a sequence of $\{1, \ldots, n\}$. Then the Travelling Salesman Problem's optimal goal can be expressed as minimizing the following objective function that can be interpreted as energy function:

$$
E(C)=d_{s_{1} s_{n}}+\sum_{i=1}^{n-1} d_{s_{i} s_{i+1}} .
$$

where $C=\left[s_{1}, s_{2}, \ldots, s_{n}\right]$ is the travelling tour. If all the costs between any two cities are equal in both directions, i.e. $D_{T}$ is a symmetric matrix, the problem is called symmetric TSP; otherwise, it is called asymmetric [23].

Sometimes $D_{T}$ is calculated based on the coordinates of the cities that may generate real numbers. Normally $d_{i j}$ 's are rounded to integer numbers to standardize the results according 
to the standard code proposed in [23]. Generation of the distance matrix, $D_{T}$, given the coordinates is a straight forward procedure. However, the reverse process is not possible for all cases.

Suppose the coordinates are given by two vectors namely $X$ and $Y$. There are $2 n$ elements in the vectors $X$ and $Y$, while a symmetric $D_{T}$ contains $1 / 2 n \times(n-1)$ elements. Each equation can be written as:

$$
\left(x_{i}-x_{j}\right)^{2}+\left(y_{i}-y_{j}\right)^{2}=d_{i j} ; i=1, \ldots, n ; j=i+1, \ldots, n-1
$$

where $x i, y i, x j$ and $y j$ are the $i$ th and $j$ th elements in $X$ and $Y$ respectively. Therefore, solution of $n(n-1) / 2$ nonlinear equations available from $D_{T}$ to find the $2 n$ unknown coordinates in $X$ and $Y$ requires:

$2 n \leq 1 / 2 n \times(n-1)$,

or equivalently:

$n \geq 5$.

Thus, there will not be a unique solution for cases with more than 5 cities. However, a feasible solution will suffice to apply the above modification to all cases with $n \geq 5$. Note that the nonlinear equations should be solved just once to find the feasible solution for the coordinates $X$ and $Y$. It is obvious that for an asymmetric $D_{T}$ there is no solution without any extra information.

\section{Initializing and annealing schedule}

As mentioned, there are not many articles talking about the initial condition when the annealing process starts. The main idea proposed in this paper is originated from the behaviour of the metal during the annealing process. The cooling schedule is an exponential shape function of the time that can be divided into three parts. The first part is a rapidly decaying curve with a high slope in average and the last one is the ending part of the exponential function with almost frozen state. The first part should be passed as fast as possible, while complying the lower bound on the rate of cooling. And the last part has almost no significant effect on the final result. Therefore, none of these two states are of interest in this paper. The initial temperature is proposed to be selected within the middle of the curve, as shown typically in Fig. 1.


(a) Temperature decay, (b) Energy decay, (c) Entropy increase

Fig. 1. Typical behaviour of an annealing schedule; the mushy state falls in the middle; 
To assign a proxy for the temperature, we may use a ratio named $\gamma$ defined as follows: $\gamma=$ the ratio of accepted new points to the total trials.

If the ratio is high, it means that the internal energy is high enough that many of motions by the atoms in a way that reduce the energy are possible. The ratio should be close to one, say $90 \%$. Conversely, if the ratio is low, say $10 \%$, the metal is nearby to become frozen; there are not so many new structures that reduce the energy. We propose to start annealing from such an initial condition in the middle zone, i.e. a mushy or doughty state, rather than liquid or firm states.

\subsection{Initiating temperature and energy}

We have applied two different methods to initialize both the temperature and energy in the mushy state. The first one is a simple algorithm for crossover elimination and the second one is an efficient fast simple method derived from a rough behaviour of a Wise Travelling Salesman (WTS), who seeks for the next nearest city. The following subsections describe the two methods.

\subsubsection{Crossover Elimination}

In the special case of TSP, knowing that the optimal tour will not contain any intersection of the paths, a simple fast algorithm of intersection detection and elimination is applied. Starting from a randomly generated initial tour, every couple of links with crossover should be deleted and replace by swapping the two links. Figure 2 easily illustrates this idea.

To do so, without lacking generality, let's continue describing the algorithm for the case that the input data is given in terms of the co-ordinates. Based on this assumption, we assume that the co-ordinates are arranged in two vectors named $X$ and $Y$. Now, let's assume that the initial random tour is named $C_{0}$. This vector is a sequence of the city indexes:

$C_{0}=\left[1, \ldots, c_{i}, c_{i+1}, \ldots, c_{j}, c_{j+1}, \ldots\right]$.

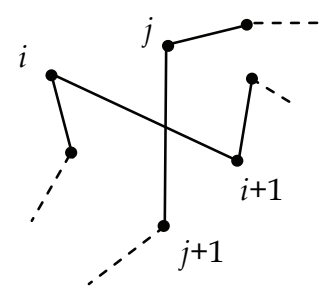

(a) $\operatorname{Tour} C_{n}$

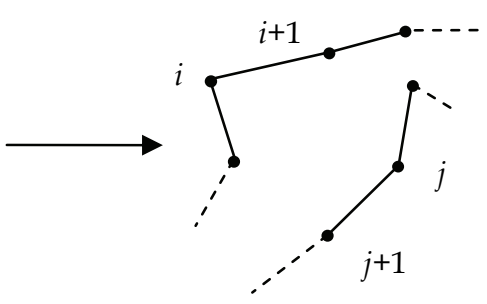

(b) Tour $C_{m}$

Fig. 2. Intersected Links Elimination

Suppose the subscripts of the elements of the sequence be the same shown in Fig. 2. Therefore, the line equations for all links of the tour can be calculated, by which it is possible to check that if each of the two paths are intersected or not. We need to solve $1 / 2 n \times(n-3)$ linear equations, where any valid solution should be in range of the coordinates $X$ and $Y$, subsequently requiring:

$x_{\min }<x_{\mathrm{c}}<x_{\max }$

$y_{\min }<y_{\mathrm{c}}<y_{\max }$ 
where, $x_{\mathrm{c}}$ and $y_{\mathrm{c}}$ are coordinates of the intersection point of each pair of links with noncommon ends, and $x_{\min }, x_{\max }, y_{\min }$ and $y_{\max }$ are the minimum and maximum coordinates found on the two links. Note that checking one of the above two conditions suffices to ensure intersection occurrence. Then, if there is a cross over, like in Fig. 2 (a), the sequence should be modified to generate $T_{m}$ as follows:

$C_{m}=\left[1, \ldots, c_{i}, c_{j}, \ldots, c_{i+1}, c_{j+1}, \ldots\right]$.

which is shown in Fig. $2(\mathrm{~b})$. It means that the $(i+1)^{\text {th }}$ element should be exchanged with the $j^{\text {th }}$ element. Then it is clear that the total cost will be reduced, i.e.:

$E\left(C_{m}\right)<E\left(C_{0}\right)$.

Once that crossover occurrence is checked $1 / 2 n \times(n-3)$ times for a tour and resolved by swapping the links, there may be new intersections generated. Therefore, the algorithm is iterated until no crossover is found in the final tour.

The algorithm is summarized in the following:

(a) Generate an initial tour randomly;

(b) Check for intersection of each path with all other non-neighbouring paths;

(c) If there is a crossover, remove it by swapping the paths;

(d) Repeat steps (b) and (c) until there is no crossover in the tour.

\subsubsection{The Wise Traveling Salesman (WTS)}

The basis thought beyond this algorithm is the way that a normal wise person may roughly decide on its next destination at each current position. For the first time that a normal human starts his/her travel, he/she may guess that a good path perhaps can continue from the nearest city. Indeed, at each step, the next city to travel is chosen among the nearest cities to the current city. Besides this original idea, one may apply a random walk process to let the traveler experience new experiments while using his/her wisdom to choose its next destination. It means that he/she examines other paths in the next experimental tour by changing some of the cities in the sequence randomly. Finally, he/she will give a weighting for his/her previously experienced tours in the next travel. However, in this paper we have not applied these two factors for initializing the SA. Thus, the simplest version of this method can be summarized in the following steps:

(a) Select the starting city randomly;

(b) Compute the cost from the current city to all unvisited cities in a vector;

(c) Sort the resulting vector elements and choose the next city with the less cost;

(d) After completing the tour, calculate the total cost (distance).

This way, the initial energy, and consequently the initial temperature when starting SA, will be much lower than what is usual.

\subsection{Annealing schedule}

The proposed method also includes modifications to the annealing schedule to compensate side effects of shortening the annealing process. The first modification is to repeat generation of new points at each temperature until the acceptance is $A_{\text {Max. The maximum }}$ iterations, sometimes called the Markov chain length, is initially considered such that $A_{\mathrm{Max}}=$ $100 \times n$, where $n$ is number of the cities in TSP. Since acceptance probability exponentially decreases, at each temperature we may reduce $A_{\mathrm{Max}}$ by a constant ratio, say 0.9 . However, in 
very low temperatures the acceptance probability is very low and there should be a criterion to agree that it is the freezing temperature. In this research, if the total iterations at each temperature, i.e. the Markov chain length, exceed $A_{\mathrm{Max}} \times n$, we stop the annealing process and call the current temperature as the freezing point.

Furthermore, in a normal SA, if the optimum solution is lost once that the algorithm faces an uphill acceptance there is enough opportunity to recover it in the future steps. When starting the annealing from low temperatures the expected time to find the global optima is shorter. Therefore, the first modification is a memory of the latest minimum energy solution found during all steps passed in the annealing. The best tour found is saved and inserted once to the process at each temperature to be compared with the current situation. This will resolve the probability of missing the best previously obtained solution.

This way the annealing process is summarized to the following steps:

(a) Employing a simple locally optimizing algorithm, set the initial temperature to a mushy state temperature, where the acceptance ratio, $\gamma$, is about $10 \%$;

(b) Set maximum acceptance to $A_{\text {Max }}=100 \times n$;

(c) Start annealing as usual, while:

i. Changing $A_{\text {Max }}$ to $0.9 \times A_{\text {Max; }}$

ii. Saving the best solution found up to now;

iii. Inserting the best solution to each Markov chain;

(d) Stop annealing if the current Markov chain length is greater than $A_{\operatorname{Max}} \times n$.

\section{The Results}

The proposed initializing method, so called Mushy State Simulated Annealing (MSSA), is applied to many benchmarks listed in TSPLIB [23]. MSSA is run 40 times: 20 runs with an initial condition obtained by crossover elimination and 20 runs initialized by WTS. The initial conditions, i.e. the initial pseudo-temperature and the initial energy are case dependent parameters. As mentioned in Section 4, we used the ratio of accepted motions (new points), which are found based on an acceptance function like (5), to the total number of tried motions $(\gamma)$. If this ratio tends to zero, the case is in its solid state, and if it is very high (near 1), then it is in the liquid state.

Figure 3 shows relation between the pseudo-temperature and the acceptance-trial ratio for the case of eli51. It is seen that in high temperatures the ratio saturates to about 1 , and in low temperatures the case has reached to its solid state with the minimum energy. Therefore, the best initial condition to start annealing is a temperature close to the melting point or the take off point in the curve, which is about the pseudo-temperature of 30 in this case.

The results for cases with below 1432 cities are given below in Table 1, through which it will be easy to realize that the method has improved SA significantly. The optimal values given by the TSBLIB site, for each case are listed in the second column of the table. We have compared the best, the worst and the average of the error in the results obtained by the new approach with other results given by recent novel works (if the best and/or the worst cases are available). The error percentage is calculated by:

$\delta=100\left(E-E^{*}\right) / E^{*}$

where $E^{*}$ is the optimal (minimum) energy. 


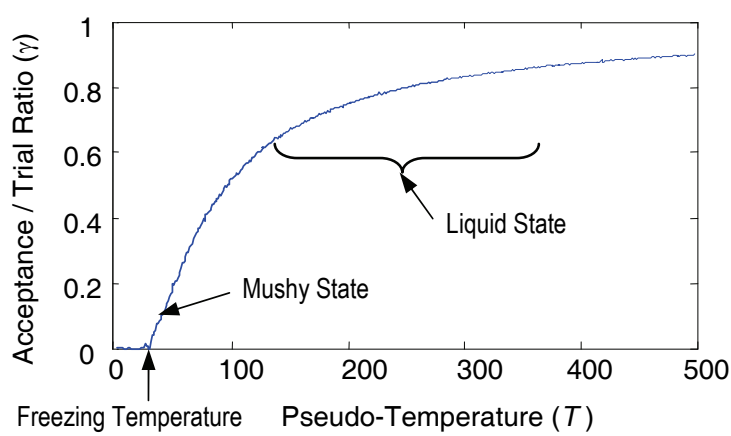

Fig. 3. Relation between the Pseudo-Temperature and the Acceptance-Trial Ratio, for the case of eli51.

The first method chosen for comparison is the Constructive Optimizing Neural Network (CONN) proposed in [24], for which it is claimed that all runs has led to the same results, so that the best, the worst and the average of the solutions are the same. The second one is a Kohonen-Like decomposition method [25], in its three different versions abbreviated by KD, KL and KG. The third is a Genetic Algorithm-Based Clustering [26]. Four variants of this method are introduced and tested, the results of which are given as EER, SE, ECER and SP. The fourth collection of the methods compared in the table are categorized under the column entitles Self-Organizing neural networks. Four versions are given in the table namely KNIES-global, KNIES-local, Budinich and ESOM [27]. The results for the normal SA are also taken from the same reference. A set of enhanced methods called Self-Organizing Map designed by Genetic Algorithms is the fifth set. There also four columns quoted for this category from [16][28]. Finally, we have compared our results with the best and the average error percentages of the results given in [29] for its memetic neural network.

It is easy to deduce that MSSA by both initializing methods has led to very accurate results, with slightly weaker characteristics for WTS as a cost of speeding up the algorithm. The proposed method has shown superiority to all other competing methods, though they are not tested for the last benchmark, u1432, which perhaps will lead to more inaccurate results, if tested. To accomplish our comparison, we have added another set of methods from [8], in which 11 methods are run on 30 benchmarks from lin105 to u1432. For brevity purpose, the problems are categorized into 3 groups, namely: small, medium and large size benchmarks. The results are given in Table 2, where the average of the average error in each group is shown. For detailed explanation of each method see [8].

\begin{tabular}{|c|c|c|c|c|}
\hline \multirow{2}{*}{ Algorithms in [7] } & \multicolumn{3}{|c|}{ Average Error in } & \multirow{2}{*}{$\begin{array}{l}\text { Total Average } \\
\text { Error }\end{array}$} \\
\hline & Small Size & Medium Size & Large Size & \\
\hline SA (Simulated Annealing) & 2.76 & 3.25 & 3.7 & 3.09 \\
\hline TA (Threshold Accepting) & 5.37 & 4.18 & 9.95 & 5.75 \\
\hline RRT (Record-to-Record Travel) & 4.22 & 6.79 & 13.96 & 6.78 \\
\hline
\end{tabular}




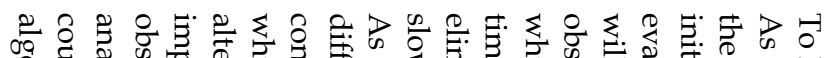

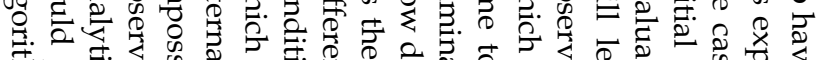

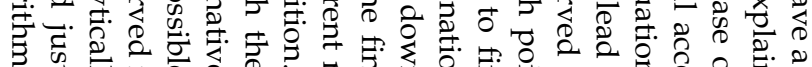



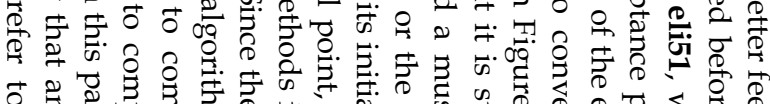
ठ



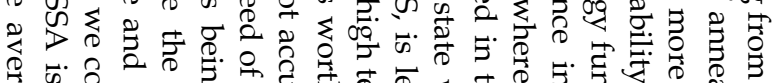



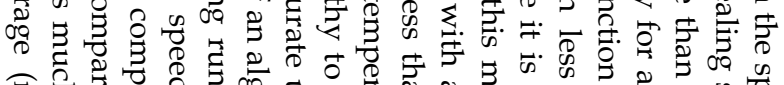



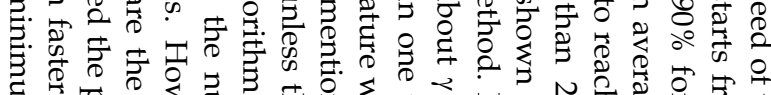

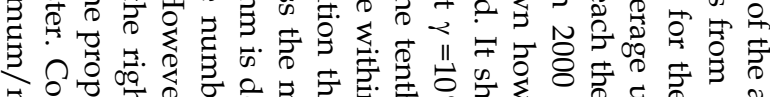

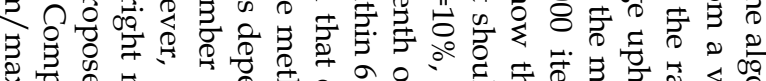

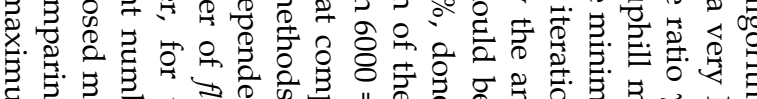

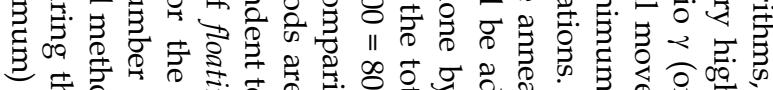

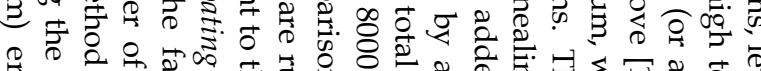

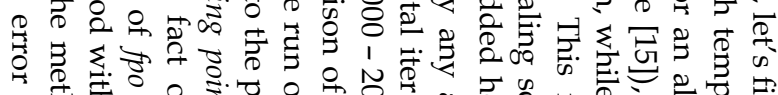



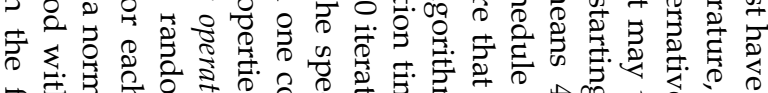



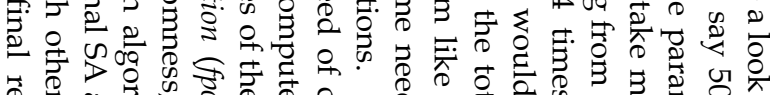

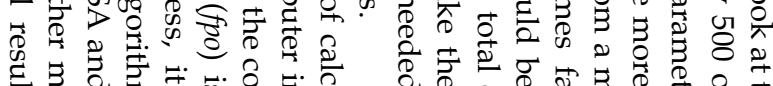

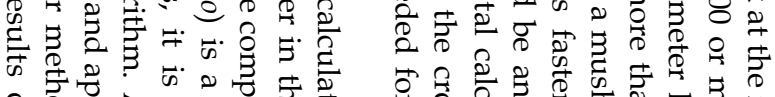

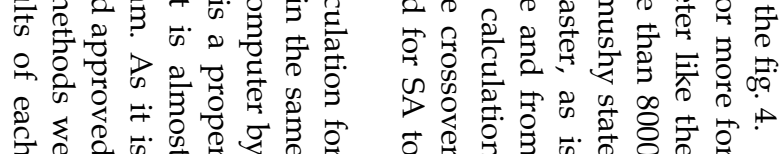

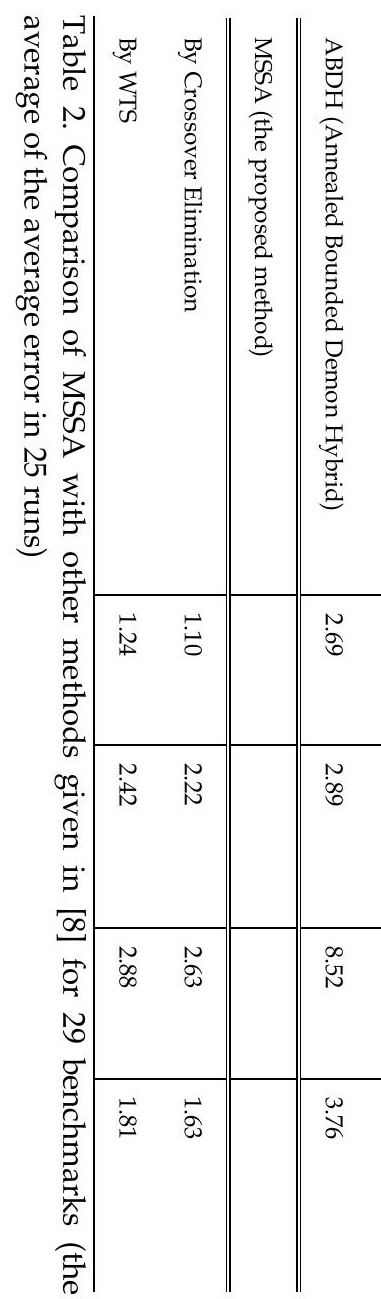




\begin{tabular}{|c|c|c|c|c|c|c|c|c|c|c|c|c|c|c|c|c|c|c|c|c|}
\hline \multirow[b]{2}{*}{ 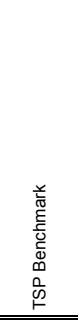 } & \multirow[b]{2}{*}{  } & \multicolumn{3}{|c|}{$\begin{array}{l}\text { MSSA By Crossover } \\
\text { Elimination }\end{array}$} & \multicolumn{3}{|c|}{ MSSA By WTS } & \multicolumn{3}{|c|}{ CONN [24] } & \multicolumn{3}{|c|}{$\begin{array}{l}\text { KOHONEN-LIKE } \\
\text { Decomposition [25] } \\
\text { (Average } \delta)\end{array}$} & \multicolumn{4}{|c|}{$\begin{array}{l}\text { Genetic Algorithm-Based } \\
\text { Clustering [26] } \\
\text { (Average } \delta)\end{array}$} & \multicolumn{3}{|c|}{$\begin{array}{r}\text { Self-Organizing neural } n \\
\text { (Average } \delta)\end{array}$} \\
\hline & & $\begin{array}{l}\infty \\
\ddot{\infty} \\
\Phi \\
\Phi\end{array}$ & 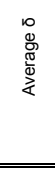 & $\begin{array}{l}\infty \\
\vec{\omega} \\
\vdots \\
\vdots\end{array}$ & $\begin{array}{l}\infty \\
\mathbb{\infty} \\
\infty \\
\infty\end{array}$ & 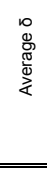 & $\begin{array}{l}\infty \\
+\infty \\
0 \\
\vdots \\
\vdots\end{array}$ & $\begin{array}{l}\infty \\
\mathbb{\infty} \\
\Phi\end{array}$ & 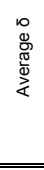 & $\begin{array}{l}\infty \\
+\infty \\
\vdots \\
\vdots\end{array}$ & $\underline{2}$ & $\overrightarrow{\vec{v}}$ & $\stackrel{\text { Oִ }}{\check{Y}}$ & 孚 & 山 & 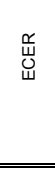 & क & 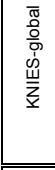 &  & ふ \\
\hline eil51 & 426 & 0 & 0.36 & 0.94 & 0 & 0.79 & 1.88 & 2.58 & 2.58 & 2.58 & 3.50 & 2.86 & 2.86 & 1.16 & 7.19 & 0.23 & 0.23 & 2.86 & 2.86 & 2.33 \\
\hline st70 & 675 & 0 & 1.02 & 1.63 & 0 & 0.90 & 1.48 & 2.96 & 2.96 & 2.96 & 3.67 & 1.51 & 2.33 & - & - & - & - & 2.33 & 1.51 & 2.14 \\
\hline eil76 & 538 & 0 & 0.82 & 1.67 & 0 & 0.70 & 2.04 & 5.02 & 5.02 & 5.02 & 6.49 & 4.98 & 5.48 & 4.27 & 3.41 & 0.92 & 2.18 & 5.48 & 4.98 & 5.54 \\
\hline gr96 & 514 & 0 & 0.83 & 1.36 & 0 & 0.53 & 1.17 & 3.61 & 3.61 & 3.61 & - & - & - & 10.62 & 8.07 & 3.09 & 2.46 & - & - & 4.12 \\
\hline kroA100 & 21282 & 0 & 0.52 & 0.93 & 0.28 & 0.65 & 2.04 & 2.57 & 2.57 & 2.57 & - & - & - & 16.54 & 6.70 & 3.81 & 2.41 & - & - & 5.94 \\
\hline rd100 & 7910 & 0.01 & 1.40 & 1.96 & 0.01 & 1.42 & 3.12 & 3.59 & 3.59 & 3.59 & 4.89 & 2.09 & 2.62 & 11.10 & 8.93 & 3.23 & 3.81 & 2.62 & 2.09 & 3.26 \\
\hline eil101 & 629 & 0 & 0.90 & 1.91 & 0 & 1.97 & 3.34 & 4.61 & 4.61 & 4.61 & 6.84 & 4.66 & 5.63 & 17.99 & 8.71 & 4.55 & 4.12 & 5.63 & 4.66 & 5.74 \\
\hline $\operatorname{lin} 105$ & 14379 & 0 & 0.52 & 1.00 & 0 & 0.33 & 0.77 & 0.38 & 0.38 & 0.38 & 2.18 & 1.98 & 1.29 & 22.55 & 2.85 & 2.31 & 3.10 & 1.29 & 1.98 & 1.87 \\
\hline pr107 & 44303 & 0 & 0.14 & 0.30 & 0 & 0.13 & 0.30 & 2.77 & 2.77 & 2.77 & 10.83 & 0.73 & 0.42 & 20.46 & 5.79 & 2.11 & 2.98 & 0.42 & 0.73 & 1.54 \\
\hline pr124 & 59030 & 0 & 0.25 & 0.60 & 0 & 0.21 & 0.45 & 1.74 & 1.74 & 1.74 & 3.22 & 0.08 & 0.49 & 30.51 & 3.75 & 2.93 & 3.02 & 0.49 & 0.08 & 1.26 \\
\hline bier127 & 118282 & 0.12 & 0.37 & 0.68 & 0.04 & 0.58 & 1.12 & 2.45 & 2.45 & 2.45 & 5.82 & 2.76 & 3.08 & 9.49 & 4.39 & 3.56 & 2.21 & 3.08 & 2.76 & 3.52 \\
\hline pr136 & 96772 & 0.35 & 1.05 & 1.97 & 0.55 & 1.43 & 2.89 & 2.27 & 2.27 & 2.27 & 1.93 & 4.53 & 5.15 & 26.50 & 12.54 & 12.43 & 6.19 & 5.15 & 4.53 & 4.90 \\
\hline gr137 & 698 & 0.14 & 0.63 & 1.29 & 0 & 0.92 & 2.00 & 4.69 & 4.69 & 4.69 & - & - & - & 23.85 & 6.58 & 2.54 & 4.22 & - & - & 8.45 \\
\hline kroA150 & 26524 & 1.36 & 2.18 & 3.37 & 0.48 & 1.47 & 2.22 & 4.78 & 4.78 & 4.78 & - & - & - & 29.15 & 7.10 & 8.01 & 5.17 & - & - & - \\
\hline kroA200 & 29368 & 0.48 & 1.22 & 2.50 & 1.03 & 1.49 & 2.30 & 4.40 & 4.40 & 4.40 & 5.66 & 5.72 & 6.57 & 40.97 & 10.46 & 7.72 & 5.91 & 6.57 & 5.72 & 5.61 \\
\hline pr226 & 80369 & 0.90 & 1.48 & 2.85 & 1.34 & 1.96 & 2.21 & 1.93 & 1.93 & 1.93 & - & - & - & 57.09 & 5.52 & 4.71 & 5.05 & - & - & - \\
\hline pr264 & 49135 & 0.85 & 2.91 & 5.55 & 1.66 & 2.83 & 4.49 & 3.58 & 3.58 & 3.58 & - & - & - & 54.08 & 10.26 & 10.49 & 9.37 & - & - & - \\
\hline $\operatorname{lin} 318$ & 42029 & 0.53 & 1.34 & 1.90 & 0.70 & 1.79 & 2.56 & - & - & - & - & - & - & 53.18 & 12.06 & 12.49 & 12.99 & - & - & 7.56 \\
\hline rd400 & 15281 & 1.90 & 2.48 & 2.85 & 2.30 & 2.54 & 2.80 & 5.77 & 5.77 & 5.77 & - & - & - & 56.83 & 17.25 & 14.33 & 15.95 & - & - & - \\
\hline
\end{tabular}




\begin{tabular}{|c|c|c|c|c|c|c|c|c|}
\hline  & $\overline{\bar{y}}$ & 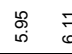 & $\underset{0}{\sigma}$ & . &  & 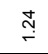 & 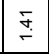 & \\
\hline î & ָָ & $\begin{array}{ll}9 \\
9 \\
6\end{array}$ &  & . & 莫 & @ִ & $\begin{array}{l} \\
\\
\end{array}$ & \\
\hline 包 & ק & $1 \bar{g}$ & $\begin{array}{l}\overline{+} \\
\dot{+}\end{array}$ & . & $\stackrel{\Delta}{N}$ & 雚 & \begin{tabular}{|l}
$\stackrel{\leftrightarrow}{\circ}$ \\
$\stackrel{2}{\circ}$ \\
\end{tabular} & \\
\hline$\underset{i s}{i}$ & 营 & . & ì & . & ָั & $\stackrel{\widetilde{c}}{\sim}$ & \begin{tabular}{|l|}
$\stackrel{\leftrightarrow}{\leftrightarrow}$ \\
\end{tabular} & $\dot{8}$ \\
\hline 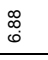 & 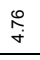 & & 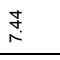 & ' & 品 & $\stackrel{\sim}{\sim}$ & \begin{tabular}{|c|}
$\stackrel{\leftrightarrow}{\circ}$ \\
\end{tabular} & $\sum_{\infty}^{\infty}$ \\
\hline $\bar{m}$ & $\begin{array}{l}\bar{\infty} \\
\dot{s} \\
\end{array}$ & $\begin{array}{r}8 \\
1 \\
0 \\
\end{array}$ & 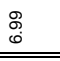 & . & $\underset{\substack{\infty \\
\infty}}{\infty}$ & $\stackrel{\sim}{\sim}$ & \begin{tabular}{|l|}
$\stackrel{\circ}{\circ}$ \\
\end{tabular} & ठ \\
\hline 等 & 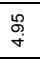 & $\begin{array}{l} \\
1 \\
1 \\
\end{array}$ & 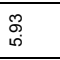 & . & ลิ & $\begin{array}{l}g \\
\\
\end{array}$ & 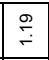 & $\stackrel{0}{\frac{0}{2}}$ \\
\hline  & $\begin{array}{l}\hat{0} \\
\hat{i} \\
\end{array}$ & 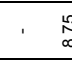 & $\begin{array}{l}\stackrel{\mathscr{N}}{\infty} \\
\infty\end{array}$ & . & \begin{tabular}{l}
\multirow{+}{+}{} \\
\\
\end{tabular} & $\begin{array}{l}\text { g. } \\
\end{array}$ & \begin{tabular}{|l|}
$\stackrel{?}{\leftarrow}$ \\
\end{tabular} & $\underbrace{\infty}_{0}$ \\
\hline$\stackrel{0}{\circ}$ & $\begin{array}{l}\infty \\
\infty \\
0 \\
\infty \\
\end{array}$ & $\begin{array}{l}0 \\
1 \\
0 \\
\end{array}$ & $\begin{array}{l}0 \\
0 \\
0\end{array}$ & . & $\underset{8}{+}$ & : & \begin{tabular}{|l|}
$\stackrel{9}{\leftarrow}$ \\
\end{tabular} & 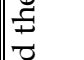 \\
\hline 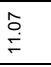 & 袋 & . & ' & . & $\underset{\substack{\infty \\
\infty}}{\infty}$ & $\begin{array}{l} \\
0 \\
0\end{array}$ & $\stackrel{2}{+}$ & 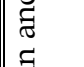 \\
\hline $\begin{array}{l}\text { ?of } \\
\stackrel{9}{0} \\
\end{array}$ & $\begin{array}{l}0 \\
0 \\
0 \\
\end{array}$ & , ' & ' & ' & $\begin{array}{l}8 \\
\dot{+}\end{array}$ & 犃 & \begin{tabular}{|l|}
$\stackrel{2}{\circ}$ \\
\end{tabular} & $\mid \begin{array}{l}3 \\
.9 \\
.9\end{array}$ \\
\hline 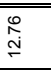 & 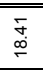 & \begin{tabular}{l}
\multirow{N}{*}{} \\
$\sim$
\end{tabular} & ' & . & $\begin{array}{l}\text { t. } \\
0 \\
\end{array}$ & 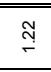 & 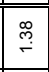 &  \\
\hline $\begin{array}{l}\stackrel{\infty}{\circ} \\
\stackrel{\rho}{\circ} \\
\end{array}$ & $\stackrel{\infty}{\stackrel{\infty}{0}}$ & 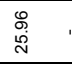 & . & . & $\stackrel{8}{\circ}$ & $\underset{\text { กิ }}{\text { กิ }}$ & \begin{tabular}{|l|}
$\stackrel{\infty}{\sim}$ \\
\end{tabular} & 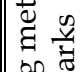 \\
\hline$\underset{+}{\bar{\Phi}}$ &  & \begin{tabular}{ll}
\multirow{2}{\infty}{} \\
$\stackrel{\infty}{\circ}$ \\
$\stackrel{5}{\circ}$
\end{tabular} & ' & . & ঙฺุ & $\stackrel{\text { ฐ̃ }}{\underline{T}}$ & \begin{tabular}{|l|}
$\stackrel{\infty}{\longrightarrow}$ \\
\end{tabular} & \\
\hline 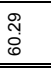 &  &  & ' & . & 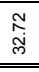 & 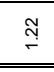 & 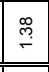 & 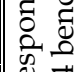 \\
\hline 然 & 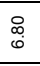 & $\begin{array}{l}0 \\
0 \\
0\end{array}$ & $\stackrel{8}{\circ}$ & . & \begin{tabular}{|l|}
\multirow{8}{*}{} \\
$\dot{+}$ \\
\end{tabular} & $\stackrel{+}{\circ}$ & \begin{tabular}{|l|}
$\stackrel{\sim}{\sim}$ \\
\end{tabular} & \\
\hline 矛 & 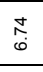 & ' & . & ' & \begin{tabular}{l|}
$\infty$ \\
$\infty$ \\
$\infty$
\end{tabular} & 苟 & \begin{tabular}{|l|}
$\stackrel{n}{*}$ \\
\end{tabular} & 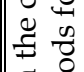 \\
\hline $\begin{array}{l}8 \\
\infty \\
\infty \\
\end{array}$ & $\begin{array}{l}\frac{6}{6} \\
\\
\end{array}$ & $\stackrel{\Sigma}{\sigma}$ & 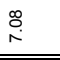 & ' & $\begin{array}{l}8 \\
i \\
\\
\end{array}$ & 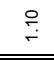 &  & 氜 \\
\hline 吕 & $\begin{array}{l}8 \\
5 \\
\end{array}$ & 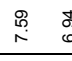 & $\begin{array}{l}\text { 苟 } \\
0 \\
0\end{array}$ & ' & \begin{tabular}{|l|}
8 \\
$\dot{+}$ \\
\end{tabular} & 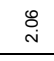 & \begin{tabular}{|c|}
$\stackrel{m}{\sim}$ \\
\end{tabular} & \\
\hline 品 & $\stackrel{8}{\circ}$ &  & $\begin{array}{l}\text { d. } \\
\text { o. } \\
0\end{array}$ & ' & \begin{tabular}{|l|} 
\\
$\dot{+}$ \\
\end{tabular} & $\stackrel{m}{\stackrel{m}{\sim}}$ & $\stackrel{f}{\stackrel{f}{\leftarrow}}$ & $\mid \begin{array}{ll}4 & 0 \\
0 & 0 \\
0 & 0\end{array}$ \\
\hline  & 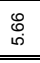 & $\begin{array}{lll}\stackrel{\circ}{\circ} & 5 \\
\wedge & 0 \\
\end{array}$ & 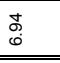 & . & \begin{tabular}{|l|}
\multirow{8}{*}{+} \\
\multirow{4}{*}{}
\end{tabular} & 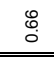 & 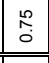 & \\
\hline 总 & लि & $\begin{array}{ll}8 & 9 \\
\text { f } & 9\end{array}$ &  & \begin{tabular}{l|l||}
\multirow{+}{\infty}{} \\
\multirow{+}{*}{}
\end{tabular} &  & $\stackrel{\text { i⿱ }}{\mathrm{i}}$ & ' & है \\
\hline ঙ̊. & ָั & 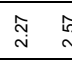 & $\stackrel{5}{\text { i }}$ & $\stackrel{\infty}{\sim}$ & 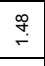 &  & . & $\underset{\infty}{\infty}$ \\
\hline$\stackrel{\infty}{\leftarrow}$ &  & 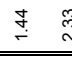 &  & 产 &  & $\begin{array}{l}: \\
\vdots \\
\end{array}$ & , & \\
\hline  & $\stackrel{\tilde{\rho}}{i}$ & 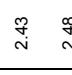 & $\stackrel{\infty}{\mathrm{N}}$ & 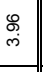 &  & & 芯 & \\
\hline & $\stackrel{8}{-}$ & $\stackrel{\circ}{\circ}$ & Ṅ & $\stackrel{\stackrel{8}{N}}{\text { N }}$ & $\stackrel{\infty}{\stackrel{\infty}{+}}$ & & 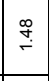 & $\begin{array}{lll}0 & \\
0 & 0 \\
0 & 0 & 0\end{array}$ \\
\hline 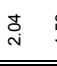 & $\stackrel{\leftrightarrow}{\leftrightarrow}$ & 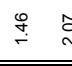 &  & $\begin{array}{l}\text { ì } \\
\text { in }\end{array}$ & $\begin{array}{l}\circ \\
\circ \\
\end{array}$ & &  & $\begin{array}{lll}3 & 0 \\
0 & \pi \\
0 & 2\end{array}$ \\
\hline 㐘 & 品 & 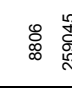 & 导 & | & 这 & 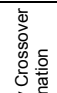 &  & 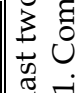 \\
\hline 管 & 篣 &  & $\begin{array}{l}\approx \\
\text { ò }\end{array}$ & 挙 & 离 & 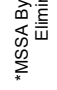 & \begin{tabular}{|l|} 
\\
\multicolumn{1}{c}{} \\
0 \\
$\sum_{*}^{n}$ \\
\end{tabular} & 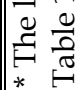 \\
\hline
\end{tabular}





(a) Normal Simulated Annealing, (b) Mushy State Simulated Annealing

Fig. 4. Energy decay in the annealing process for eli51;

\section{Conclusion}

Simulated annealing is one of the top ten methods of non-derivative based optimization methods, various versions of which are proposed by researchers during the two last decades. Focusing on the initial condition by which the annealing starts, this paper proposes a novel variant of the original SA named mushy state simulated annealing (MSSA). In this method we start annealing not from a high temperature in a liquid state, but from a low temperature in a mushy state. Moreover, we use a memory to save the best solution found previously. This technique has speeded up the optimization process while achieving to quite accurate optimum solutions. For the case study of TSP, two simple algorithms including crossover elimination and the shortly introduced method of WTS are used to initiate the MSSA. Results are compared to many recent new optimization methods that are applied to solve TSP. Despite of its higher speed compared to the normal SA, superiority of the proposed method is observed in all cases with less than 1432 cities. The average error obtained by MSSA for the 24 benchmarks is much less than all other methods compared to this method.

\section{References}

[1] S. Kirkpatrick, C. D. Gelatt, and M. P. Vecchi, “Optimization by simulated annealing," Science, vol. 220, pp. 671-680, 1983.

[2] M. R. Garey and D. S. Johnson, "Computers and Intractability: A Guide to the Theory of NP-Completeness", New York: Freeman, 1979.

[3] Herbert H. Tsang, and Kay C. Wiese "The Significance of Thermodynamic Models in the Accuracy Improvement of RNA Secondary Structure Prediction Using Permutation-based Simulated Annealing", IEEE Congress on Evolutionary Computation (CEC), 2007.

[4] Ming-Hao Hung, Li-Sun Shu, Shinn-Jang Ho, Shiow-Fen Hwang, and Shinn-Ying Ho, “A Novel Intelligent Multiobjective Simulated Annealing Algorithm for Designing Robust PID Controllers", IEEE Transactions on Systems, Man, and Cybernetics PART A: Systems and Humans, Vol. 38, No. 2, pp. 319-330, March 2008. 
[5] Kevin I. Smith, Richard M. Everson, Jonathan E. Fieldsend, Chris Murphy, and Rashmi Misra, "Dominance-Based Multiobjective Simulated Annealing", IEEE Transactions On Evolutionary Computation, vol. 12, no. 3, pp. 323-341, June 2008.

[6] S. A. Kravitz and R. A. Rutenbar, "Placement by simulated annealing on a multiprocessor," IEEE Transactions Computer-Aided Design Integr. CircuitsSyst., vol. 6, no. 4, pp. 534-549, Jul. 1987.

[7] E. Aarts and J. Korst, "Simulated Annealing and Boltzmann Machines: A Stochastic Approach to Combinatorial Optimization and Neural Computing", New York: Wiley, 1989.

[8] Joshua W. Pepper, Bruce L. Golden, and Edward A. Wasil, "Solving the Traveling Salesman Problem With Annealing-Based Heuristics: A Computational Study", IEEE Transactions on Systems, Man, and Cybernetics - PART A: Systems and Humans, Vol. 32, No. 1, Jan. 2002.

[9] Hyeon-Joong Cho, Se-Young Oh and Doo-Hyun Choi, "Population-oriented simulated annealing technique based on local Temperature concept", Electronics Letters, vol. 34, no. 3, pp.312-313, 5th February 1998.

[10] Percy P. C. Yip, and Yoh-Han Pao, "Combinatorial Optimization with Use of Guided Evolutionary Simulated Annealing", IEEE Transactions on Neural Networks, vol. 6, no. 2, pp. 290-295, March 1995.

[11] Andrew Soh, "Parallel N-ary Speculative Computation of Simulated Annealing", IEEE Transactions on Parallel and Distributed Systems, vol. 6, no. 10, pp. 997-1005, October 1995.

[12] D. C. W. Pao, S. P. Lam and A. S. Fong, "Parallel implementation of simulated annealing using transaction processing", IEE Proc-Comput. Digit. Tech.. Vol. 146, No. 2, March 1999, pp. 107-113.

[13] Feng-Tse Lin, Cheng-Yan Kao, and Ching-Chi Hsu, "Applying the Genetic Approach to Simulated Annealing in Solving Some NP-Hard Problems", IEEE Transactions on Systems, Man, and Cybernetics, Vol. 23. No. 6, Nov./Dec. 1993.

[14] Dale R. Thompson and Griff L. Bilbro, "Sample-Sort Simulated Annealing", IEEE Transactions on Systems, Man, and Cybernetics - PART B: Cybernetics, Vol. 35, No. 3, pp. 625-632 Jun. 2005.

[15] Hao Chen, Nicholas S. Flann, and Daniel W. Watson, "Parallel Genetic Simulated Annealing: A Massively Parallel SIMD Algorithm", IEEE Transactions on Parallel and Distributed Systems, vol. 9, no. 2, pp.126-136, February 1998.

[16] K.L. Wong A.G.Constantinides, "Speculative parallel simulated annealing with acceptance prediction", Electronics Letters, vol. 34, no. 3, pp. 312-313, 5th February 1998.

[17] L. Ingber and B. Rosen, "Genetic Algorithms and Very Fast Simulated Reannealing: A Comparison," Mathematical Computer Modeling, vol. 16, no. 11, pp. 87-100, 1992.

[18] Lipo Wang, Sa Li, Fuyu Tian, and Xiuju Fu, “A Noisy Chaotic Neural Network for Solving Combinatorial Optimization Problems: Stochastic Chaotic Simulated Annealing", Transactions on Systems, Man, and Cybernetics-PART B: Cybernetics, Vol. 34, No. 5 pp. 2119-2125, Oct. 2004.

[19] Yuyao He, "Chaotic Simulated Annealing With Decaying Chaotic Noise", IEEE Transactions on Neural Networks, vol. 13, no. 6, pp. 1526-1531, November 2002. 
[20] Sitao Wu and Tommy W. S. Chow, "Self-Organizing and Self-Evolving Neurons: A New Neural Network for Optimization", IEEE Transactions on Neural Networks, vol. 18, no. 2, pp. 385-396, March 2007.

[21] J. Jang, C. Sun, E. Mizutani, "Neuro-Fuzzy and Soft Computing", Proc. of the Prentice Hall 1997.

[22] G. Dueck and T. Scheuer, "Threshold accepting: A general purpose optimization algorithm appearing superior to simulated annealing," J. Computer. Phys., vol. 90, 1990, pp. 161-175.

[23] G. Reinelt. Tsplib95, 1995. Available at: http://www.iwr.uni-heidelberg.de/groups/comopt/ software/TSPLIB95.

[24] M. Saadatmand-Tarzjan, M. Khademi, M. R. Akbarzadeh-T., and H. Abrishami Moghaddam, "A Novel Constructive-Optimizer Neural Network for the Traveling Salesman Problem" IEEE Transactions on Systems, Man, and Cybernetics-PART B: Cybernetics, Vol. 37, No. 4, Aug. 2007.

[25] Necati Aras, I. Kuban Altınel, and John Oommen, "A KOHONEN-LIKE DECOMPOSITION METHOD FOR THE EUCLIDEAN TRAVELING SALESMAN PROBLEM KNIES_DECOMPOSE", IEEE Transactions on Neural Networks, vol. 14, no. 4, July 2003.

[26] Chun-Hung Cheng, Wing-Kin Lee, and Kam-Fai Wong, "A Genetic Algorithm-Based Clustering Approach for Database Partitioning", IEEE Transactions on Systems, Man, and Cybernetics - PART C: Applications and Reviews, Vol. 32, No. 3, Aug. 2002.

[27] Kwong-Sak Leung, Hui-Dong Jin, and Zong-Ben Xu, "An expanding Self-Organizing neural network for the traveling salesman problem", Neurocomputing, Vol. 62, pp. 267-292, Dec. 2004.

[28] Hui-Dong Jin, Kwong-Sak Leung, Man-Leung Wong and Zong-Ben Xu, “An Efficient Self-Organizing Map Designed by Genetic Algorithms for the Traveling Salesman Problem", IEEE Transactions on Systems, Man, and Cybernetics - PART B: Cybernetics, Vol. 33, No. 6, pp. 877-888, Dec. 2003.

[29] J. C. Creput, A. Koukam, "A memetic neural network for the Euclidean traveling salesman problem", Neurocomputing Accepted 22, January 2008. 
Simulated Annealing.

Theory with Applications

estes by hui chibants

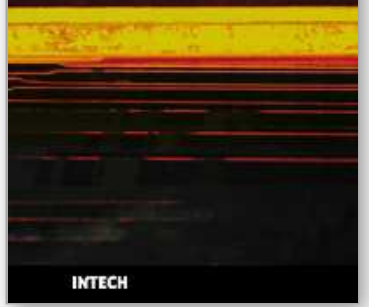

\section{Simulated Annealing, Theory with Applications}

Edited by Rui Chibante

ISBN 978-953-307-134-3

Hard cover, 292 pages

Publisher Sciyo

Published online 18, August, 2010

Published in print edition August, 2010

The book contains 15 chapters presenting recent contributions of top researchers working with Simulated Annealing (SA). Although it represents a small sample of the research activity on SA, the book will certainly serve as a valuable tool for researchers interested in getting involved in this multidisciplinary field. In fact, one of the salient features is that the book is highly multidisciplinary in terms of application areas since it assembles experts from the fields of Biology, Telecommunications, Geology, Electronics and Medicine.

\section{How to reference}

In order to correctly reference this scholarly work, feel free to copy and paste the following:

Kambiz Shojaee G., Hamed Shakouri G. and Mojtaba Behnam Taghadosi (2010). Importance of the Initial Conditions and the Time Schedule in the Simulated Annealing, Simulated Annealing, Theory with Applications, Rui Chibante (Ed.), ISBN: 978-953-307-134-3, InTech, Available from:

http://www.intechopen.com/books/simulated-annealing--theory-with-applications/importance-of-the-initialconditions-and-the-time-schedule-in-the-simulated-annealing

\section{INTECH}

open science | open minds

\section{InTech Europe}

University Campus STeP Ri

Slavka Krautzeka 83/A

51000 Rijeka, Croatia

Phone: +385 (51) 770447

Fax: +385 (51) 686166

www.intechopen.com

\section{InTech China}

Unit 405, Office Block, Hotel Equatorial Shanghai

No.65, Yan An Road (West), Shanghai, 200040, China

中国上海市延安西路65号上海国际贵都大饭店办公楼 405 单元

Phone: +86-21-62489820

Fax: $+86-21-62489821$ 
(C) 2010 The Author(s). Licensee IntechOpen. This chapter is distributed under the terms of the Creative Commons Attribution-NonCommercialShareAlike-3.0 License, which permits use, distribution and reproduction for non-commercial purposes, provided the original is properly cited and derivative works building on this content are distributed under the same license. 